\title{
Prevalence of metabolic dysfunction-associated fatty liver disease \& its association with physical function in patients with acute coronary syndrome
}

\author{
Takumi Noda \\ Kitasato University: Kitasato Daigaku \\ Kentaro Kamiya ( $\sim$ k-kamiya@kitasato-u.ac.jp) \\ Nobuaki Hamazaki \\ Kitasato University Hospital: Kitasato Daigaku Byoin \\ Kohei Nozaki \\ Kitasato University Hospital: Kitasato Daigaku Byoin \\ Takafumi Ichikawa \\ Kitasato University Hospital: Kitasato Daigaku Byoin \\ Masashi Yamashita \\ Kitasato University: Kitasato Daigaku \\ Shota Uchida \\ Kitasato University: Kitasato Daigaku

\section{Emi Maekawa} \\ Kitasato University School of Medicine: Kitasato Daigaku Igakubu \\ Tasuku Terada \\ University of Ottawa Heart Institute \\ Jennifer Reed \\ University of Ottawa Heart Institute \\ Minako Yamaoka-Tojo \\ Kitasato University: Kitasato Daigaku \\ Atsuhiko Matsunaga \\ Kitasato University: Kitasato Daigaku \\ Junya Ako \\ Kitasato University School of Medicine: Kitasato Daigaku Igakubu
}

\section{Research}

Keywords: metabolic dysfunction-associated fatty liver disease, acute coronary syndrome, liver dysfunction, frailty, sarcopenia, physical function

Posted Date: February 10th, 2022

DOI: https://doi.org/10.21203/rs.3.rs-1268673/v1

License: (c) (i) This work is licensed under a Creative Commons Attribution 4.0 International License. Read Full License 


\section{Abstract}

Background and Aims: It is widely known that patients with acute coronary syndrome (ACS) are at increased risk of nonalcoholic fatty liver disease (NAFLD), which is linked to sarcopenia and physical dysfunction. However, the relationship between metabolic dysfunction-associated fatty liver disease (MAFLD) and physical dysfunction remains unclear. The purpose of this study was to investigate the prevalence of MAFLD in patients with ACS to assess the relationship between MAFLD and muscle strength, walking speed, and 6-minute walking distance (6 MWD).

Methods and Results: We reviewed patients with ACS who were assessed for hepatic steatosis using the fatty liver index, and the results were further assessed to determine the presence of MAFLD. Multiple regression analysis was used to examine the association between MAFLD and physical function. Among 479 enrolled hospitalized patients, MAFLD was identified in 234 (48.9\%) patients. We conducted a multiple regression analysis in which MAFLD was independently associated with lower leg strength, gait speed, and 6 MWD (leg strength, $P=0.023 ;$ gait speed, $P=0.002$ and $6 \mathrm{MWD}, \mathrm{P}=0.017)$. Furthermore, having more metabolic impairment was still associated with lower physical function decline (leg strength, $\mathrm{P}$ for trend $=0.002$; gait speed, $\mathrm{P}$ for trend $=0.019$ and 6 MWD, $\mathrm{P}$ for trend $=0.003$ ).

Conclusions: MAFLD is common in hospitalized patients with ACS, and most patients with MAFLD have many overlapping metabolic abnormalities. MAFLD is associated with impaired physical function, and the greater the number of overlapping metabolic abnormalities, the worse the motor function.

Registration number: KMEO B18-075

\section{Introduction}

Nonalcoholic fatty liver disease (NAFLD) is recognized as the most prevalent chronic liver disease worldwide, with an estimated global prevalence of $20-30 \%$ [1-3]. NAFLD is a multisystem disease that affects organs and regulatory pathways other than the liver [4-7]. Liver fibrosis caused by NAFLD progression is also associated with increased mortality $[8,9]$. Furthermore, NAFLD is more common in patients with cardiovascular diseases (CVD) $[2,5,10,11]$, including patients with acute coronary syndrome (ACS), with this condition increasing the risk of recurring CVD events. Therefore, the relationship between NAFLD and cardiovascular events has attracted attention for CVD prevention $[3,10]$. Recently, a new name and definition for NAFLD has been proposed to better understand fatty liver [12]; this new name is metabolic dysfunctionassociated fatty liver disease (MAFLD). The newly proposed definition is based on the coexistence of hepatic fat deposition and overweight/obesity, diabetes mellitus (DM), or multiple metabolic abnormalities and can be diagnosed regardless of daily alcohol consumption or other liver diseases [12]. Therefore, it has been proposed that MAFLD is a more appropriate term to describe liver disease with a background of metabolic abnormalities. Metabolic diseases, such as obesity and DM, are risk factors for developing ACS [13, 14], and ischemic heart disease (IHD) patients often present with several metabolic risk factors. Thus, the prevalence of MAFLD in patients with ACS may be higher than in the general population due to the combined presence of metabolic risk factors such as DM and obesity in this population despite an increased prevalence of these risk factors in the general population too.

MAFLD in patients with ACS is also expected to be more likely to result in decreased physical function. Previous studies have demonstrated that NAFLD in patients with CVD has often been associated with muscle weakness or reduced walking speed due to insulin resistance and chronic inflammation [15]. The combination of NAFLD and physical dysfunction is associated with poor prognosis and decreased quality of life (QOL) [16-18]. However, it is unclear whether MAFLD in patients with ACS is involved in declining physical functions such as lower leg strength and walking speed. Thus, this study aimed to investigate the prevalence of MAFLD in patients with ACS and to clarify the relationship between MAFLD and physical dysfunction.

\section{Methods}

\section{Study Population}

A total of 479 patients with ACS admitted to the Kitasato University Hospital Cardiovascular Center between May 2008 and November 2018 were included in this cross-sectional study. All patients were defined as having acute ST-segment elevation, non-ST-segment elevation myocardial infarction, or unstable angina diagnosed according to American College of Cardiology/American Heart Association guidelines [19] and were assessed for the presence of hepatic steatosis by fatty liver index (FLI). Patients undergoing maintenance hemodialysis were excluded from the study. The study protocol was designed according to the tenets of the Declaration of Helsinki and approved by the Ethics Committee of the Kitasato University Medical Ethics Organization (KMEO) (no. KMEO B18-075). Since the present study is an observational study that did not involve invasive procedures or interventions, written informed consent was not required according to the principles set forth in the "Ethical Guidelines for Medical and Health Research for Subjects" by the Japanese Ministry of Health, Labor and Welfare. Therefore, informed consent was waived by the KMEO according to the institutional guidelines for retrospective observational studies. All participants were informed that they could choose to withdraw at any point or refuse to participate in the study. 


\section{Data collection and assessment of MAFLD}

The clinical characteristics of the patients (age, sex, body mass index [BMI], underlying disease [angina pectoris or myocardial infarction], laboratory examination results, vital signs (blood pressure and heart rate), echocardiographic data, etc.) were collected from the electronic medical records at the time of discharge. Additionally, clinical information (comorbidities and medication use) at discharge were recorded.

We assessed the presence of hepatic steatosis in each patient using the fatty liver index (FLI) [20, 21], which was calculated using the formula

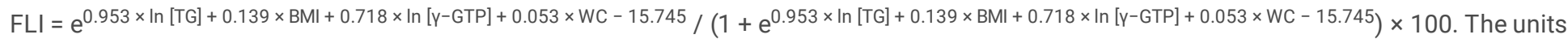
for triglyceride (TG), gamma-glutamyl transpeptidase ( $y-G T P)$, and waist circumference (WC) were $\mathrm{mg} / \mathrm{L}, \mathrm{U} / \mathrm{L}$, and cm, respectively. Furthermore, according to Yang et al. [4], hepatic steatosis was defined as FLI $\geq 35$ for men and FLI $\geq 20$ for women.

MAFLD was diagnosed based on FLI-confirmed hepatic steatosis with any of the three metabolic conditions: DM, overweight/obesity, or at least two metabolic risk abnormalities. According to the MAFLD definition [12], metabolic risk abnormalities were defined as the presence of the following criteria: 1) WC $\geq 90 \mathrm{~cm}$ in men and $80 \mathrm{~cm}$ in women, 2) prediabetes (hemoglobinA1c 5.7 to $6.4 \%$ ), 3) blood pressure $\geq 130 / 85 \mathrm{mmHg}$ or under antihypertensive therapy, 4) high-density lipoprotein cholesterol (HDL-C) $<40 \mathrm{mg} / \mathrm{dL}$ in men and $<50 \mathrm{mg} / \mathrm{dL}$ in women, 5) TG $\geq 150$ $\mathrm{mg} / \mathrm{dL}$ or specific drug treatment, and 6) C-reactive protein (CRP) level $>2 \mathrm{mg} / \mathrm{L}$. The homeostasis model assessment-insulin resistance (HOMA-IR) score was not investigated in this study.

\section{Physical function tests}

Physical function tests such as leg strength, gait speed, and 6-minute walking distance (6 MWD) were measured before discharge. Leg strength was defined as the maximal isometric quadriceps muscle strength and was measured using a portable dynamometer ( $\mu$-Tas; ANIMA, Tokyo, Japan). The detailed measurement method and reliability are described in our previous study (AJM). Briefly, the patient was seated in a chair, and the maximum isometric voluntary contraction of the quadriceps muscle was measured for $5 \mathrm{~s}$, twice in each leg with the hip joint flexed approximately at $90^{\circ}$ using a non-extensible strap connected to a strain gauge at the ankle. Measurements were performed on the right and left quadriceps in succession. Left and right maximal muscle strength values were averaged and expressed as absolute values ( $\mathrm{kg}$ ) and relative to body mass (\%BM).

Usual gait speed was measured when the participants were asked to walk for $10 \mathrm{~m}$ in the middle of a $16 \mathrm{~m}$ sidewalk at their average pace. The normal walking rate of each participant was calculated by dividing the distance $(\mathrm{m})$ by the time $(\mathrm{s})$.

The 6 MWD was measured under the supervision of a technician according to the guidelines of the American Thoracic Society (ATS) [22]. Patients were instructed to walk at their own pace along a straight, flat corridor marked at 1-m intervals, and the distance (in $\mathrm{m}$ ) was recorded after 6 min.

\section{Statistical Analysis}

The results for distributed continuous data are presented as medians (interquartile range). Categorical variables were expressed as numbers and percentages. The patients were divided into two groups: those with MAFLD and those without MAFLD. The baseline characteristics were compared using the Mann-Whitney $\mathrm{U}$ test for continuous variables and the chi-square test for categorical variables, as appropriate. Nonnormally distributed variables were transformed to a logarithmic scale for analysis. Multiple imputations using R with the "mice" package version 3.13 .0 [23] generated 20 datasets with complemented missing values.

We used the Cochran-Armitage trend test to analyze the relationship between the prevalence of MAFLD and age. We also used Euler diagrams (area-proportional diagrams) to visualize the number of patients in the disjoint and overlapping areas of the three metabolic conditions domains using $\mathrm{R}$ with the "eulerr" package version 6.1 .0 (https://cran.r-project.org/package=eulerr).

To evaluate the strength of the association between leg strength, gait speed, $6 \mathrm{MWD}$, and MAFLD, we used multiple linear regression models adjusted for the following variables: age, sex, BMI, log maximum creatine kinase (CK), number of diseased vessels, statin use, aspirin use, prior heart failure, hypertension, dyslipidemia, DM, smoking, log albumin, and log hemoglobin. In addition, multiple linear regression analysis adjusted for the same variables was performed to assess the association of MAFLD with leg strength, gait speed, and 6 MWD when stratified by the total number of the three metabolic conditions, that is, DM, overweight/obesity, or metabolic risk abnormalities.

All statistical analyses were performed using R Studio statistical software (version 3.6.2; R: A language and environment for statistical computing, R Core Team, R Foundation for Statistical Computing, Vienna, Austria, 2019, https://www.R-project.org). The level of statistical significance was set at $P<0.05$.

\section{Results}


The baseline characteristics of the study participants are shown in Table 1 . The median age of the study population was 65 years, with $80.8 \%$ men and 19.2\% women. The median lower extremity muscle strength, gait speed, and $6 \mathrm{MWD}$ of the study participants were $47.4 \% \mathrm{BM}$, $1.2 \mathrm{~m} / \mathrm{s}$, and $486 \mathrm{~m}$, respectively. 


\begin{tabular}{|c|c|c|c|c|}
\hline & Overall & Non-MAFLD & MAFLD & \\
\hline & $\mathrm{n}=479$ & $\mathrm{n}=245 ; 51.1 \%$ & $n=234 ; 48.9 \%$ & P-value \\
\hline Age [years] & $65[56-73]$ & $69[61-74]$ & $63[52-70]$ & $<0.001$ \\
\hline Male, n (\%) & $387(80.8)$ & $205(83.7)$ & $182(77.8)$ & 0.128 \\
\hline $\mathrm{BMI}\left[\mathrm{kg} / \mathrm{m}^{2}\right]$ & $23.4[21.5-25.7]$ & $21.8[20.3-23.0]$ & $25.5[23.9-27.7]$ & $<0.001$ \\
\hline Heart rate [beats/min] & $72[65-84]$ & $71[64-82]$ & $75[65-86]$ & 0.049 \\
\hline Systolic blood pressure [mm Hg] & 114 [101 - 133] & $113[100-130]$ & $117[103-135]$ & 0.068 \\
\hline Diastolic blood pressure [mm Hg] & $66[59-77]$ & $65[58-74]$ & $68[61-80]$ & 0.001 \\
\hline \multicolumn{5}{|l|}{ Diagnosis, n (\%) } \\
\hline Myocardial infarction & $438(91.4)$ & $226(92.2)$ & $212(90.6)$ & 0.631 \\
\hline Angina & $41(8.6)$ & $19(7.8)$ & $22(9.4)$ & 0.631 \\
\hline \multicolumn{5}{|l|}{ Treatment, n (\%) } \\
\hline $\mathrm{PCl}$ & $412(86.0)$ & $209(85.3)$ & $203(86.8)$ & 0.746 \\
\hline CABG & $32(6.7)$ & $19(7.8)$ & $13(5.6)$ & 0.435 \\
\hline Number of diseased vessels, $n(\%)$ & & & & 0.252 \\
\hline 1 & $204(47.1)$ & $97(43.9)$ & $107(50.5)$ & \\
\hline 2 & $147(33.9)$ & $83(37.6)$ & $64(30.2)$ & \\
\hline 3 & $82(18.9)$ & $41(18.6)$ & $41(19.3)$ & \\
\hline LVEF [\%] & $53.0[45.0-60.0]$ & $52.0[44.5-61.2]$ & $53.6[45.0-60.0]$ & 0.920 \\
\hline Prior heart failure, n (\%) & $26(5.4)$ & $16(6.5)$ & $10(4.3)$ & 0.374 \\
\hline Waist circumference [cm] & $87.0[81.5-93.0]$ & $82.5[78.0-87.0]$ & $92.5[87.8-97.5]$ & $<0.001$ \\
\hline \multicolumn{5}{|l|}{ Comorbidities } \\
\hline Hypertension, n (\%) & $375(78.3)$ & $183(74.7)$ & $192(82.1)$ & 0.066 \\
\hline Dyslipidemia, n (\%) & $539(68.1)$ & $251(60.2)$ & $288(76.8)$ & $<0.001$ \\
\hline Diabetes mellitus, n (\%) & $256(53.4)$ & $123(50.2)$ & $133(56.8)$ & 0.173 \\
\hline Obesity, n (\%) & $158(33.0)$ & $18(7.3)$ & $140(59.8)$ & $<0.001$ \\
\hline Current smoker, n (\%) & $178(38.0)$ & $82(34.2)$ & $96(41.9)$ & 0.102 \\
\hline \multicolumn{5}{|l|}{ Medications } \\
\hline Beta Blocker, n (\%) & $423(88.3)$ & $214(87.3)$ & $209(89.3)$ & 0.597 \\
\hline ACE inhibitor or ARB, $n(\%)$ & $433(90.4)$ & $222(90.6)$ & $211(90.2)$ & 0.993 \\
\hline Statin, n (\%) & 451 (94.2) & $236(96.3)$ & 215 (91.9) & 0.060 \\
\hline Aspirin, n (\%) & $319(66.6)$ & $163(66.5)$ & $156(66.7)$ & 0.999 \\
\hline \multicolumn{5}{|l|}{ Laboratory examination } \\
\hline $\mathrm{CRP}[\mathrm{mg} / \mathrm{dL}]$ & $0.40[0.10-1.10]$ & $0.40[0.10-1.00]$ & $0.40[0.20-1.20]$ & 0.639 \\
\hline Triglyceride [mg/dL] & $114[89-149]$ & $98[78-123]$ & 139 [107 - 174] & $<0.001$ \\
\hline Total cholesterol & $143[126-163]$ & 137 [123 - 159] & $146[132-167]$ & 0.003 \\
\hline LDL-C [mg/dL] & $82[67-98]$ & $78[65-97]$ & $84[72-100]$ & 0.006 \\
\hline HDL-C [mg/dL] & $39[33-46]$ & $41[34-50]$ & $37[32-43]$ & $<0.001$ \\
\hline
\end{tabular}




\begin{tabular}{|c|c|c|c|c|}
\hline & Overall & Non-MAFLD & MAFLD & \\
\hline Total bilirubin [mg/dL] & $0.5[0.4-0.7]$ & $0.5[0.4-0.6]$ & $0.5[0.4-0.7]$ & 0.006 \\
\hline AST [U/L] & $22[17-29]$ & $21[17-27]$ & $22[18-31]$ & $<0.001$ \\
\hline $\mathrm{ALT}[\mathrm{U} / \mathrm{L}]$ & $24[16-37]$ & $21[15-31]$ & $28[20-45]$ & $<0.001$ \\
\hline Y-GTP & $34[23-53]$ & $27[20-42]$ & $45[29-82]$ & $<0.001$ \\
\hline Albumin [g/dL] & $3.8[3.4-4.1]$ & $3.7[3.3-4.0]$ & $3.9[3.5-4.1]$ & 0.002 \\
\hline Hemoglobin [g/dL] & $13.1[12.0-14.3]$ & $12.8[11.7-14.1]$ & $13.4[12.3-14.6]$ & $<0.001$ \\
\hline $\mathrm{HbA} 1 \mathrm{c}[\%]$ & $6.0[5.5-6.7]$ & $5.8[5.4-6.5]$ & $6.1[5.6-6.8]$ & $<0.001$ \\
\hline Maximum CK [U/L] & $2122[1014-3839]$ & 2041 [952 - 3683] & 2279 [1075 - 4198] & 0.238 \\
\hline Fatty liver index [point] & $33.0[17.4-54.2]$ & $17.7[11.5-26.6]$ & $55.1[42.8-68.9]$ & $<0.001$ \\
\hline \multicolumn{5}{|l|}{ Physical function } \\
\hline Leg strength [\%BM] & $47.4[37.1-61.7]$ & $47.6[37.8-63.5]$ & $47.0[37.0-58.9]$ & 0.226 \\
\hline Gait speed $[\mathrm{m} / \mathrm{s}]$ & $1.2[1.0-1.4]$ & $1.2[1.1-1.4]$ & $1.2[1.0-1.3]$ & 0.891 \\
\hline 6-minute walking distance [m] & $486[405-544]$ & $483[405-540]$ & $489[406-550]$ & 0.534 \\
\hline \multicolumn{5}{|c|}{ Median [interquartile range]; n, number (\%) } \\
\hline \multicolumn{5}{|c|}{$\begin{array}{l}\text { MAFLD, metabolic dysfunction-associated fatty liver disease; BMI, body mass index; PCI, percutaneous intracoronary intervention; CRBG, } \\
\text { coronary aortic bypass graft; LVEF, left ventricular ejection fraction; ACE, angiotensin-converting enzyme; ARB, angiotensin receptor blocker } \\
\text { CRP, C-reactive protein; LDL-C, low-density lipoprotein cholesterol; HDL-C, high-density lipoprotein cholesterol; AST, aspartate } \\
\text { aminotransferase; ALT, alanine aminotransferase; } \mathrm{Y}-\mathrm{GTP} \text {, gamma-glutamyl transpeptidase; HbA1c, hemoglobinA1C, CK, creatine kinase. }\end{array}$} \\
\hline
\end{tabular}

According to the predefined definition, 234 patients (48.9\%) had MAFLD. As shown in Figure 1, MAFLD was more common in younger patients and was found in $65.8 \%, 48.6 \%, 34.1 \%$, and $31.3 \%$ of patients under 60 years, in their $60 \mathrm{~s}$, in their $70 \mathrm{~s}$, and 80 years and older, respectively ( $P$ for trend < 0.001). In the Euler diagram shown in Figure 2, 198 (41.3\%), 230 (48.0\%), and 135 (28.2\%) patients had disorders in the domains of overweight/obesity, metabolic risk abnormalities, and DM, respectively. Furthermore, there was a significant overlap in the three metabolic conditions, and the most common combination was having all metabolic disorders (24.0\%). Baseline characteristics were stratified by the presence or absence of MAFLD. MAFLD was associated with younger age, male sex, higher BMI, indicators of obesity, and higher liver function tests (such as aspartate aminotransferase [AST], alanine aminotransferase [ALT], and $\mathrm{Y}-\mathrm{GTP}$ ).

Table 2 summarizes the multivariate linear regression analysis results used to assess the association between physical function (leg strength, gait speed, and $6 \mathrm{MWD}$ ) and MAFLD. MAFLD was an independent determinant of each physical function, even after adjustment for covariates related to the severity of ACS and poor physical function (leg strength, $\beta:-0.110, P=0.023$, gait speed, $\beta:-0.151, P=0.002$ and $6 \mathrm{MWD}, \beta:$ $0.111, P=0.017$ ). Furthermore, MAFLD was also associated with lower physical function (Figure 3 ). We also conducted the same analyses to evaluate the effects of multiple metabolic disorders on physical dysfunction (Figure 4). After adjusting for the same covariates, having more metabolic condition impairments in MAFLD patients was still associated with lower physical function decline (leg strength, $\mathrm{P}$ for trend $=0.024$, gait speed, $\mathrm{P}$ for trend $=0.010$, and $6 \mathrm{MWD}, \mathrm{P}$ for trend $=0.035$ ). 
Table 2

Associations of MAFLD with physical function tests

\begin{tabular}{|c|c|c|c|c|c|c|c|c|c|c|c|c|}
\hline \multirow[b]{2}{*}{ Effect } & \multicolumn{4}{|c|}{ Leg strength } & \multicolumn{4}{|l|}{ Gait speed } & \multicolumn{4}{|c|}{ 6-minute walking distance } \\
\hline & $\begin{array}{l}\text { B } \\
\text { coefficient }\end{array}$ & $\beta$ & $\begin{array}{l}\mathrm{t} \\
\text { value }\end{array}$ & $\begin{array}{l}\mathrm{P}- \\
\text { value }\end{array}$ & $\begin{array}{l}\text { B } \\
\text { coefficient }\end{array}$ & $\beta$ & $\begin{array}{l}\mathrm{t} \\
\text { value }\end{array}$ & $\begin{array}{l}\mathrm{P}- \\
\text { value }\end{array}$ & $\begin{array}{l}\text { B } \\
\text { coefficient }\end{array}$ & $\beta$ & $\begin{array}{l}\mathrm{t} \\
\text { value }\end{array}$ & $\begin{array}{l}\mathrm{P} \text { - } \\
\text { value }\end{array}$ \\
\hline MAFLD & -3.888 & -0.110 & -2.289 & 0.023 & -0.089 & -0.151 & -3.046 & 0.002 & -26.388 & -0.111 & -2.403 & 0.017 \\
\hline Age & -0.453 & -0.318 & -6.698 & $<0.001$ & -0.008 & -0.326 & -6.918 & $\begin{array}{l}< \\
0.001\end{array}$ & -4.262 & -0.423 & -9.754 & $<.001$ \\
\hline Sex (male) & 11.21 & 0.256 & 6.292 & $\begin{array}{l}< \\
0.001\end{array}$ & 0.081 & 0.119 & 2.535 & 0.001 & 64.843 & 0.210 & 5.332 & $\begin{array}{l}<.001 \\
0.01\end{array}$ \\
\hline BMI & -0.470 & -0.086 & -1.874 & 0.062 & 0.007 & 0.079 & 1.656 & 0.098 & 0.532 & 0.014 & 0.324 & 0.746 \\
\hline $\begin{array}{l}\text { Log } \\
\text { maximum } \\
\text { CK }\end{array}$ & 0.308 & 0.013 & 0.418 & 0.676 & 0.005 & 0.006 & 0.415 & 0.678 & -1.672 & -0.017 & -0.326 & 0.745 \\
\hline $\begin{array}{l}\text { Number of } \\
\text { diseased } \\
\text { vessels }\end{array}$ & 0.555 & 0.021 & 0.589 & 0.556 & -0.012 & -0.037 & -0.673 & 0.501 & -11.514 & -0.074 & -1.784 & 0.076 \\
\hline Statin & -1.757 & -0.021 & -0.608 & 0.544 & -0.012 & -0.007 & -0.218 & 0.828 & 19.763 & 0.043 & 0.957 & 0.340 \\
\hline Aspirin & 4.890 & 0.130 & 3.216 & 0.001 & 0.071 & 0.115 & 2.717 & 0.007 & 23.836 & 0.096 & 2.354 & 0.019 \\
\hline $\begin{array}{l}\text { Prior heart } \\
\text { failure }\end{array}$ & -0.183 & -0.004 & -0.064 & 0.949 & -0.021 & -0.013 & -0.420 & 0.675 & 11.383 & 0.023 & 0.613 & 0.540 \\
\hline Hypertension & -1.935 & -0.052 & -1.191 & 0.234 & 0.003 & 0.005 & 0.107 & 0.915 & -6.418 & -0.023 & -0.617 & 0.538 \\
\hline Dyslipidemia & 2.759 & 0.067 & 1.922 & 0.055 & -0.008 & -0.023 & -0.318 & 0.751 & -6.287 & -0.024 & -0.657 & 0.512 \\
\hline $\begin{array}{l}\text { Diabetes } \\
\text { mellitus }\end{array}$ & -3.213 & -0.090 & -2.420 & 0.016 & -0.012 & -0.021 & -0.528 & 0.598 & -15.021 & -0.063 & -1.712 & 0.088 \\
\hline Smoking & -1.301 & -0.034 & -0.883 & 0.378 & -0.024 & -0.041 & -0.950 & 0.343 & -10.648 & -0.039 & -1.098 & 0.273 \\
\hline Log Alb & 17.606 & 0.145 & 3.135 & 0.002 & 0.343 & 0.171 & 3.533 & $\begin{array}{l}< \\
0.001\end{array}$ & 206.206 & 0.241 & 5.494 & $\begin{array}{l}< \\
0.001\end{array}$ \\
\hline $\log \mathrm{Hb}$ & 14.128 & 0.113 & 2.356 & 0.019 & 0.267 & 0.132 & 2.570 & 0.011 & 88.545 & 0.101 & 2.299 & 0.022 \\
\hline
\end{tabular}

$\beta$, standardized regression coefficient.

MAFLD, metabolic dysfunction-associated fatty liver disease; BMI, body mass index; CK, creatine kinase; Alb, albumin; Hb, hemoglobin.

\section{Discussion}

This is the first study to investigate the prevalence of MAFLD, overlapping metabolic abnormalities, and their impact on physical function in patients with ACS. The significant findings of this study are as follows: (1) among patients with ACS, about half had MAFLD, (2) patients with MAFLD were more likely to be younger and had the highest proportion of the three metabolic abnormalities, (3) in patients with ACS, the presence of MAFLD was associated with lower physical functioning, and (4) the higher the number of background metabolic abnormalities, the lower the physical functioning. These findings underscore the importance of comprehensive evaluation of metabolic abnormalities and MAFLD in patients with ACS, even in younger patients.

Many studies have reported that NAFLD is more common in patients with IHD and combines many common risk factors, such as DM and obesity $[2,5,6,11]$. In this study, we defined MAFLD using the definition proposed by Eslam et al. [12] and showed that 48.9\% of patients had MAFLD. This percentage was similar to that reported in a meta-analytic study that assessed the prevalence of MAFLD in obesity (50.7\%) [24]. Therefore, the estimates obtained from our study sample may be close to its prevalence in a real-world clinical setting. 
Secondly, it is generally reported that the prevalence of NAFLD/MAFLD tends to increase with age. On the contrary, several studies that have examined the prevalence of fatty liver disease, stratified by age, have demonstrated an increasing trend up to the age of $40-50$ years but a lower prevalence after the age of 60 years [25-27]. In addition, a sharp increase in the prevalence of fatty liver disease in younger age groups, especially in Asians, has been reported $[28,29]$. In the present study, the prevalence of MAFLD was high in young people under 60 years of age and tended to decrease in older individuals. In addition, muscle weakness and decreased walking speed occurred at a high rate in patients with IHD and NAFLD [1, 30,31], and previous studies have linked these conditions [32, 33]. However, the present study is the first to report an association between MAFLD and physical function in patients with ACS. It is important to understand the relationship between these conditions since early treatment with MAFLD may lead to the prevention and treatment of sarcopenia and muscle weakness and further improvement of clinical outcomes such as cardiovascular events and death.

The association between MAFLD and physical dysfunction in patients with IHD can be attributed to several underlying mechanisms. Patients with NAFLD are often reported to develop sarcopenia; this can be attributed to the presence of insulin resistance, excess adipose tissue, and chronic low-grade inflammation in this population. [1, 6, 34]. Hepatic steatosis and the resulting NAFLD can lead to additional chronic inflammation by secreting inflammatory cytokines such as IL6, TNF-alpha, and leptin [35]. These inflammatory cytokines may also decrease the anabolic effect of insulin-like growth factor-1 and promote insulin resistance [1, 36]. It is also known that in metabolic disorders such as obesity and diabetes mellitus, insulin resistance can lead to the accumulation of ectopic lipids in muscle cells and other organs [37], and weakened muscles due to these factors lead to decreased mitochondrial function [38]. However, skeletal muscle has been reported to be an endocrine organ that secretes myokines that regulate systemic metabolism [1]. The metabolic effects of irisin, a type of myokine, have been linked to improvements in glucose metabolism and hepatic steatosis [39], and skeletal muscle loss may exacerbate these conditions. These mechanisms have also been shown in studies of patients with cardiovascular disease [40]; therefore, MAFLD in patients with ACS may be more strongly associated with decreased muscle strength, walking speed, and exercise tolerance.

MAFLD and physical dysfunction are important factors associated with CVD events and mortality [17, 41], and accurate assessment of both in hospitalized patients with ACS is necessary. These conditions are not merely indicators reflecting disease severity but are treatable; thus, improving these conditions may significantly improve clinical outcomes. A study in patients with MAFLD showed that aerobic exercise improved liver fibrosis severity and $\mathrm{VO}_{2 \max }$ [42]. In addition, studies of patients with NAFLD reported that aerobic exercise, resistance training, and nutritional therapy significantly improved liver fibrosis [43-45]. Furthermore, it has been reported that patients with MAFLD who have a greater number of underlying metabolic disorders also have significantly higher severity of liver fibrosis [46, 47]; it is widely known that cardiac rehabilitation of patients with CVD also improves their underlying metabolic disorders (DM, dyslipidemia, hypertension, etc.). Therefore, it is suggested that appropriate cardiac rehabilitation for patients with ACS may lead to improved MAFLD and prognosis.

In this study, FLI was used to assess hepatic steatosis. Biochemical data of TG and Y-GTP in patients are commonly measured in clinical practice, and WC and body weight are easily measurable, making it easy to assess hepatic steatosis in any patient. Therefore, it may be possible to evaluate MAFLD early in patients who do not have background liver disease and therefore do not have a detailed liver function examination. In the present study, about half of the patients with ACS had MAFLD, which was associated with decreased physical function. Our findings suggest that assessing MAFLD in patients with ACS may help detect an early decline in muscle strength and gait speed, allowing for more individualized decisions regarding therapeutic intervention and prevention of CVD recurrence in ACS patients.

\section{Study Limitations}

This study had some limitations. First, because this was a single-case cross-sectional study, the causal relationship between MAFLD and motor dysfunction is unknown. Furthermore, the study did not show an association between MAFLD and the prognosis of patients with IHD. Second, this study included only Asian patients with ACS. Therefore, further studies are needed to determine whether the results can be generalized to other ethnic groups. Third, in this study, hepatic steatosis was assessed using FLI, and there was no additional information on ultrasonography or imaging of the liver. However, FLI can assess fatty liver by ultrasonography with high accuracy [4, 20, 48] and has been reported to be associated with NAFLD development [49]. Furthermore, the Asian Pacific Association's guidelines for the management of MAFLD recommend using FLI to evaluate hepatic steatosis [50]. Therefore, evaluation with FLI, which is easy to perform, may help detect MAFLD early. Finally, there is limited information on DM and treatment status related to obesity and metabolic disorders in the context of MAFLD. Therefore, there is a need to further investigate the long-term disease status and changes in physical function in MAFLD.

\section{Conclusion}

In conclusion, MAFLD is common in patients with ACS, and most patients with MAFLD have many overlapping metabolic abnormalities. MAFLD is associated with impaired physical function, and the greater the number of overlapping metabolic abnormalities, the worse the motor function. Assessment and intervention of the newly defined MAFLD in patients with ACS may be an early predictor of physical functional decline and may improve life expectancy and QOL.

Page $8 / 15$ 


\section{Abbreviations}

\section{ACS}

Acute coronary syndrome

BMI

Body mass index

$\mathrm{Cl}$

Confidence interval

CVD

Cardiovascular diseases

DM

Diabetes Mellitus

FLI

Fatty liver index

$Y$ - GTP

Gamma-glutamyl transpeptidase

$\mathrm{HF}$

Heart failure

IHD

Ischemic heart disease

LVEF

Left ventricular ejection fraction

MAFLD

Metabolic dysfunction-associated fatty liver disease

NAFLD

nonalcoholic fatty liver disease

TG

Triglyceride

WC

Waist circumference

6MWD

6-minute walking distance

\section{Declarations}

Ethics Approval: The study protocol was designed according to the tenets of the Declaration of Helsinki and approved by the Ethics Committee of the Kitasato University Medical Ethics Organization (KMEO) (no. KMEO B18-075).

Patient consent statement: Since the present study is an observational study that did not involve invasive procedures or interventions, written informed consent was not required according to the principles set forth in the "Ethical Guidelines for Medical and Health Research for Subjects" by the Japanese Ministry of Health, Labor and Welfare. Therefore, informed consent was waived by the KMEO according to the institutional guidelines for retrospective observational studies.

Availability of data and materials: The datasets used and/or analysed during the current study are available from the corresponding author on reasonable request.

Conflict of Interest: The author(s) declare no potential conflicts of interest with respect to the research, authorship, and/or publication of this article.

Funding statement: This work was partially supported by JSPS KAKENHI (Grant Number 21H03309).

\section{Author Contribution}

$\mathrm{TN}, \mathrm{KK}, \mathrm{AM}$, and JA contributed to the conception and design of the study. TN, KK, NH, KN, TI, MY, SU, EM, and MT contributed to the acquisition, analysis, and interpretation of data for the work. TN and KK drafted the manuscript. NH, KN, TI, MY, SU, EM, JR, MT, AM, and JA critically revised the manuscript. All authors gave final approval and agreed to be accountable for all aspects of work, ensuring integrity and accuracy. 


\section{References}

1. Kim JA, Choi KM. Sarcopenia and fatty liver disease. Hepatol Int. 2019;13:674-87.

2. Bhatia LS, Curzen NP, Calder PC, Byrne CD. Non-alcoholic fatty liver disease: a new and important cardiovascular risk factor? Eur Heart J. 2012;33:1190-200.

3. Brunner KT, Pedley A, Massaro JM, Hoffmann U, Benjamin EJ, Long MT. Increasing liver fat is associated with progression of cardiovascular risk factors. Liver Int. 2020;40:1339-43.

4. Yang BL, Wu WC, Fang KC, Wang YC, Huo TI, Huang YH, Yang HI, Su CW, Lin HC, Lee FY, et al. External validation of fatty liver index for identifying ultrasonographic fatty liver in a large-scale cross-sectional study in Taiwan. PLoS One. 2015;10:e0120443.

5. Francque SM, van der Graaff D, Kwanten WJ. Non-alcoholic fatty liver disease and cardiovascular risk: Pathophysiological mechanisms and implications. J Hepatol. 2016;65:425-43.

6. Kumar V, Hsueh WA, Raman SV. Multiorgan, Multimodality Imaging in Cardiometabolic Disease. Circ Cardiovasc Imaging $2017,10$.

7. Byrne CD, Targher G. NAFLD: a multisystem disease. J Hepatol. 2015;62:47-64.

8. Angulo P, Kleiner DE, Dam-Larsen S, Adams LA, Bjornsson ES, Charatcharoenwitthaya P, Mills PR, Keach JC, Lafferty HD, Stahler A, et al. Liver Fibrosis, but No Other Histologic Features, Is Associated With Long-term Outcomes of Patients With Nonalcoholic Fatty Liver Disease. Gastroenterology. 2015;149:389-97.e310.

9. Hagström H, Talbäck M, Andreasson A, Walldius G, Hammar N. Repeated FIB-4 measurements can help identify individuals at risk of severe liver disease. J Hepatol. 2020;73:1023-9.

10. Stahl EP, Dhindsa DS, Lee SK, Sandesara PB, Chalasani NP, Sperling LS. Nonalcoholic Fatty Liver Disease and the Heart: JACC State-of-theArt Review. J Am Coll Cardiol. 2019;73:948-63.

11. Janssen A, Grobbee DE, Dendale P. Non-alcoholic fatty liver disease, a new and growing risk indicator for cardiovascular disease. Eur J Prev Cardiol 2019:2047487319891783.

12. Eslam M, Newsome PN, Sarin SK, Anstee QM, Targher G, Romero-Gomez M, Zelber-Sagi S, Wai-Sun Wong V, Dufour JF, Schattenberg JM, et al. A new definition for metabolic dysfunction-associated fatty liver disease: An international expert consensus statement. J Hepatol. 2020;73:202-9.

13. Einarson TR, Acs A, Ludwig C, Panton UH. Prevalence of cardiovascular disease in type 2 diabetes: a systematic literature review of scientific evidence from across the world in 2007-2017. Cardiovasc Diabetol. 2018;17:83.

14. Reaven GM. Insulin resistance: the link between obesity and cardiovascular disease. Med Clin North Am. 2011;95:875-92.

15. Kim TN, Choi KM. The implications of sarcopenia and sarcopenic obesity on cardiometabolic disease. J Cell Biochem. 2015;116:1171-8.

16. Kasper P, Martin A, Lang S, Kütting F, Goeser T, Demir M, Steffen HM. NAFLD and cardiovascular diseases: a clinical review. Clin Res Cardiol. 2021;110:921-37.

17. Moon JH, Koo BK, Kim W. Non-alcoholic fatty liver disease and sarcopenia additively increase mortality: a Korean nationwide survey. J Cachexia Sarcopenia Muscle 2021.

18. Ida S, Kojima Y, Hamaoka S, Urawa N, Araki J, Kaneko R, Murata K. Validity of Japanese version of SARC-F questionnaire in patients with chronic liver disease. J Gastroenterol Hepatol. 2019;34:947-53.

19. Anderson JL, Adams CD, Antman EM, Bridges CR, Califf RM, Casey DE Jr, Chavey WE 2nd, Fesmire FM, Hochman JS, Levin TN, et al. 2011 ACCF/AHA Focused Update Incorporated Into the ACC/AHA 2007 Guidelines for the Management of Patients With Unstable Angina/NonST-Elevation Myocardial Infarction: a report of the American College of Cardiology Foundation/American Heart Association Task Force on Practice Guidelines. Circulation. 2011;123:e426-579.

20. Bedogni G, Bellentani S, Miglioli L, Masutti F, Passalacqua M, Castiglione A, Tiribelli C. The Fatty Liver Index: a simple and accurate predictor of hepatic steatosis in the general population. BMC Gastroenterol. 2006;6:33.

21. Wong VW, Chan WK, Chitturi S, Chawla Y, Dan YY, Duseja A, Fan J, Goh KL, Hamaguchi M, Hashimoto E, et al. Asia-Pacific Working Party on Non-alcoholic Fatty Liver Disease guidelines 2017-Part 1: Definition, risk factors and assessment. J Gastroenterol Hepatol. 2018;33:70-85.

22. ATS statement: guidelines for the six-minute walk test. Am J Respir Crit Care Med 2002, 166:111-117.

23. Zhang Z. Multiple imputation with multivariate imputation by chained equation (MICE) package. Ann TransI Med. 2016;4:30.

24. Liu J, Ayada I, Zhang X, Wang L, Li Y, Wen T, Ma Z, Bruno MJ, de Knegt RJ, Cao W, et al: Estimating Global Prevalence of Metabolic Dysfunction-Associated Fatty Liver Disease in Overweight or Obese Adults. Clin Gastroenterol Hepatol 2021.

25. Tobari M, Hashimoto E. Characteristic Features of Nonalcoholic Fatty Liver Disease in Japan with a Focus on the Roles of Age, Sex and Body Mass Index. Gut Liver. 2020;14:537-45.

26. Golabi P, Paik J, Reddy R, Bugianesi E, Trimble G, Younossi ZM. Prevalence and long-term outcomes of non-alcoholic fatty liver disease among elderly individuals from the United States. BMC Gastroenterol. 2019;19:56.

Page $10 / 15$ 
27. Wang Z, Xu M, Peng J, Jiang L, Hu Z, Wang H, Zhou S, Zhou R, Hultström M, Lai EY. Prevalence and associated metabolic factors of fatty liver disease in the elderly. Exp Gerontol. 2013;48:705-9.

28. Zhou J, Zhou F, Wang W, Zhang XJ, Ji YX, Zhang P, She ZG, Zhu L, Cai J, Li H. Epidemiological Features of NAFLD From 1999 to 2018 in China. Hepatology. 2020;71:1851-64.

29. Wesolowski SR, Kasmi KC, Jonscher KR, Friedman JE. Developmental origins of NAFLD: a womb with a clue. Nat Rev Gastroenterol Hepatol. 2017;14:81-96.

30. Uchida S, Kamiya K, Hamazaki N, Matsuzawa R, Nozaki K, Ichikawa T, Suzuki Y, Nakamura T, Yamashita M, Kariya H, et al. Association between sarcopenia and atherosclerosis in elderly patients with ischemic heart disease. Heart Vessels. 2020. 10.1007/s00380-0002001554-00388.

31. Biolo G, Cederholm T, Muscaritoli M. Muscle contractile and metabolic dysfunction is a common feature of sarcopenia of aging and chronic diseases: from sarcopenic obesity to cachexia. Clin Nutr. 2014;33:737-48.

32. Laube R, Wang H, Park L, Heyman JK, Vidot H, Majumdar A, Strasser SI, McCaughan GW, Liu K. Frailty in advanced liver disease. Liver Int. 2018;38:2117-28.

33. Afilalo J, Alexander KP, Mack MJ, Maurer MS, Green P, Allen LA, Popma JJ, Ferrucci L, Forman DE. Frailty assessment in the cardiovascular care of older adults. J Am Coll Cardiol. 2014;63:747-62.

34. Shida T, Akiyama K, Oh S, Sawai A, Isobe T, Okamoto Y, Ishige K, Mizokami Y, Yamagata K, Onizawa K, et al. Skeletal muscle mass to visceral fat area ratio is an important determinant affecting hepatic conditions of non-alcoholic fatty liver disease. $\mathrm{J}$ Gastroenterol. 2018;53:535-47.

35. Ahima RS, Flier JS. Adipose tissue as an endocrine organ. Trends Endocrinol Metab. 2000;11:327-32.

36. Stanley TL, Grinspoon SK. Effects of growth hormone-releasing hormone on visceral fat, metabolic, and cardiovascular indices in human studies. Growth Horm IGF Res. 2015;25:59-65.

37. Wang M, Tan Y, Shi Y, Wang X, Liao Z, Wei P. Diabetes and Sarcopenic Obesity: Pathogenesis, Diagnosis, and Treatments. Front Endocrinol (Lausanne). 2020;11:568.

38. Cleasby ME, Jamieson PM, Atherton PJ. Insulin resistance and sarcopenia: mechanistic links between common co-morbidities. J Endocrinol. 2016;229:R67-81.

39. Boström P, Wu J, Jedrychowski MP, Korde A, Ye L, Lo JC, Rasbach KA, Boström EA, Choi JH, Long JZ, et al. A PGC1-a-dependent myokine that drives brown-fat-like development of white fat and thermogenesis. Nature. 2012;481:463-8.

40. Bielecka-Dabrowa A, Ebner N, Dos Santos MR, Ishida J, Hasenfuss G, von Haehling S. Cachexia, muscle wasting, and frailty in cardiovascular disease. Eur J Heart Fail 2020.

41. Kim D, Konyn P, Sandhu KK, Dennis BB, Cheung AC, Ahmed A. Metabolic dysfunction-associated fatty liver disease is associated with increased all-cause mortality in the United States. J Hepatol 2021.

42. O'Gorman P, Naimimohasses S, Monaghan A, Kennedy M, Melo AM, Doherty DNF, Beddy DG, Finn P, Moore SP. JB, et al: Improvement in histological endpoints of MAFLD following a 12-week aerobic exercise intervention. Aliment Pharmacol Ther. 2020;52:1387-98.

43. Słomko J, Zalewska M, Niemiro W, Kujawski S, Słupski M, Januszko-Giergielewicz B, Zawadka-Kunikowska M, Newton J, Hodges L, Kubica J, Zalewski P. Evidence-Based Aerobic Exercise Training in Metabolic-Associated Fatty Liver Disease: Systematic Review with MetaAnalysis. J Clin Med 2021, 10.

44. Katsagoni CN, Georgoulis M, Papatheodoridis GV, Panagiotakos DB, Kontogianni MD. Effects of lifestyle interventions on clinical characteristics of patients with non-alcoholic fatty liver disease: A meta-analysis. Metabolism. 2017;68:119-32.

45. Hashida R, Kawaguchi T, Bekki M, Omoto M, Matsuse H, Nago T, Takano Y, Ueno T, Koga H, George J, et al. Aerobic vs. resistance exercise in non-alcoholic fatty liver disease: A systematic review. J Hepatol. 2017;66:142-52.

46. Huang J, Ou W, Wang M, Singh M, Liu Y, Liu S, Wu Y, Zhu Y, Kumar R, Lin S. MAFLD Criteria Guide the Subtyping of Patients with Fatty Liver Disease. Risk Manag Healthc Policy. 2021;14:491-501.

47. Lee K, Sung JA, Kim JS, Park TJ. The roles of obesity and gender on the relationship between metabolic risk factors and non-alcoholic fatty liver disease in Koreans. Diabetes Metab Res Rev. 2009;25:150-5.

48. Xu Z, Li H, Tian S, Wu J, Li X, Liu ZL, Li S, Chen YL, Xiao J, Wei JY, et al. Blood biomarkers for the diagnosis of hepatic steatosis in metabolic dysfunction-associated fatty liver disease. J Hepatol. 2020;73:1264-5.

49. Motamed N, Faraji AH, Khonsari MR, Maadi M, Tameshkel FS, Keyvani H, Ajdarkosh H, Karbalaie Niya MH, Rezaie N, Zamani F. Fatty liver index (FLI) and prediction of new cases of non-alcoholic fatty liver disease: A population-based study of northern Iran. Clin Nutr. 2020;39:468-74.

50. Eslam M, Sarin SK, Wong VW, Fan JG, Kawaguchi T, Ahn SH, Zheng MH, Shiha G, Yilmaz Y, Gani R, et al. The Asian Pacific Association for the Study of the Liver clinical practice guidelines for the diagnosis and management of metabolic associated fatty liver disease. Hepatol

Page $11 / 15$ 
Int. 2020;14:889-919.

\section{Figures}

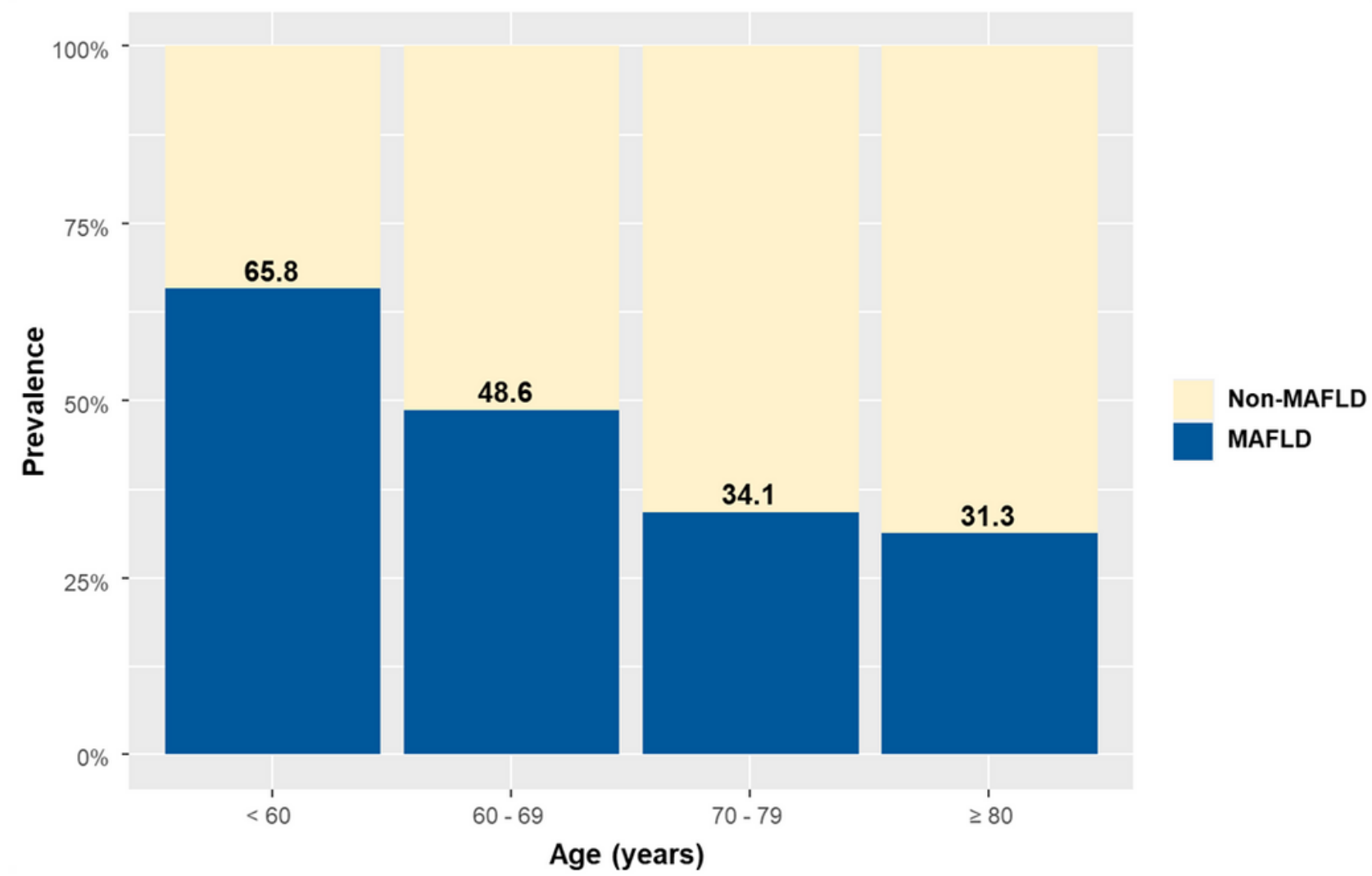

Figure 1

Prevalence of metabolic dysfunction-associated fatty liver disease in according to age category 


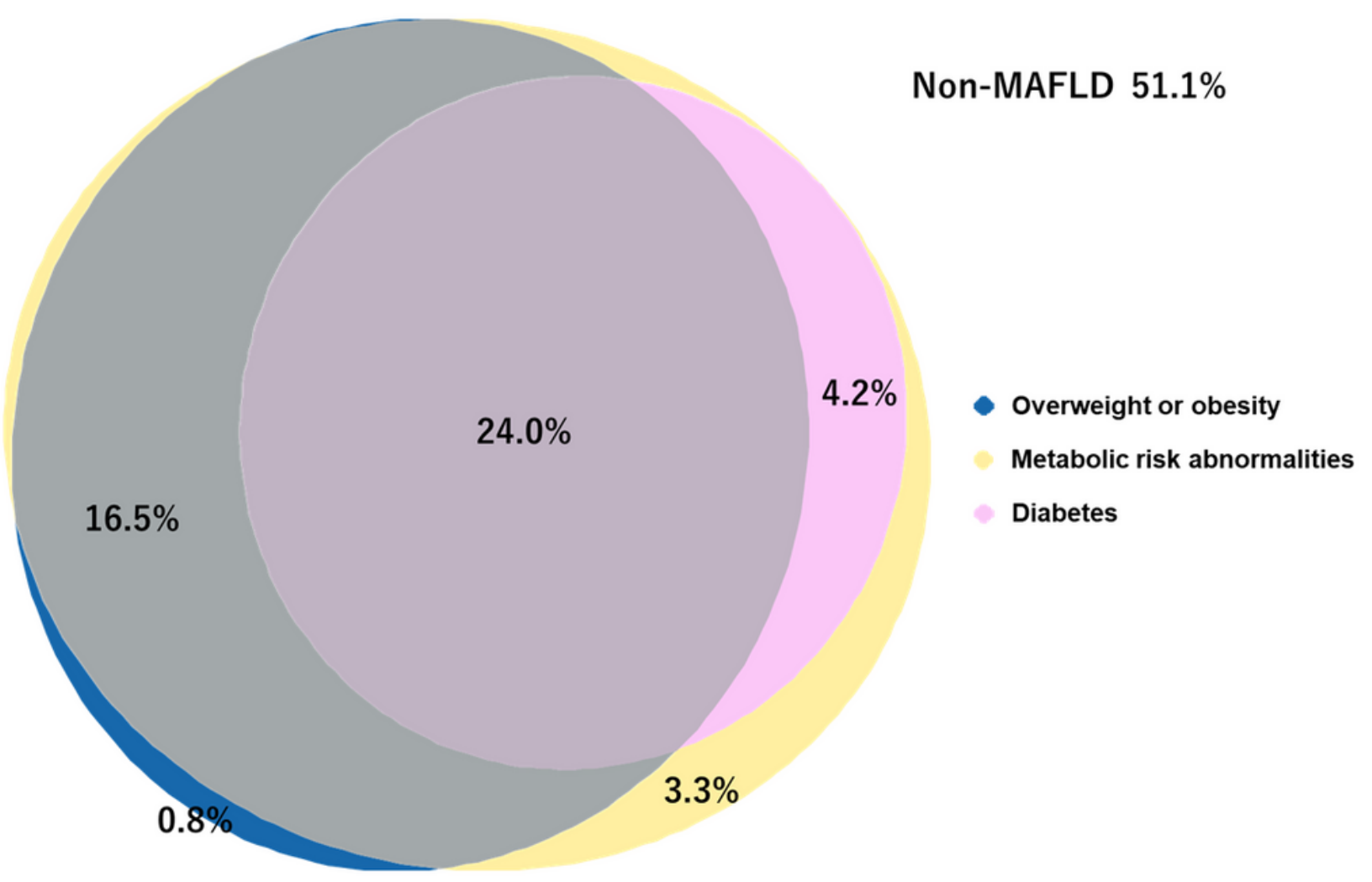

\section{Figure 2}

The proportion of overlap and non-overlap among metabolic conditions in patients with metabolic dysfunction-associated fatty liver disease (MAFLD). Euler diagrams (area-proportional diagrams) visualize the number of patients in the disjoint and overlapping three metabolic disorders. Three circles indicate the prevalence of overweight or obesity, metabolic risk abnormalities, and diabetes, respectively. The percentage of the population in the single and overlapped domains is also shown. The rate is about the total cohort $(n=479)$ 

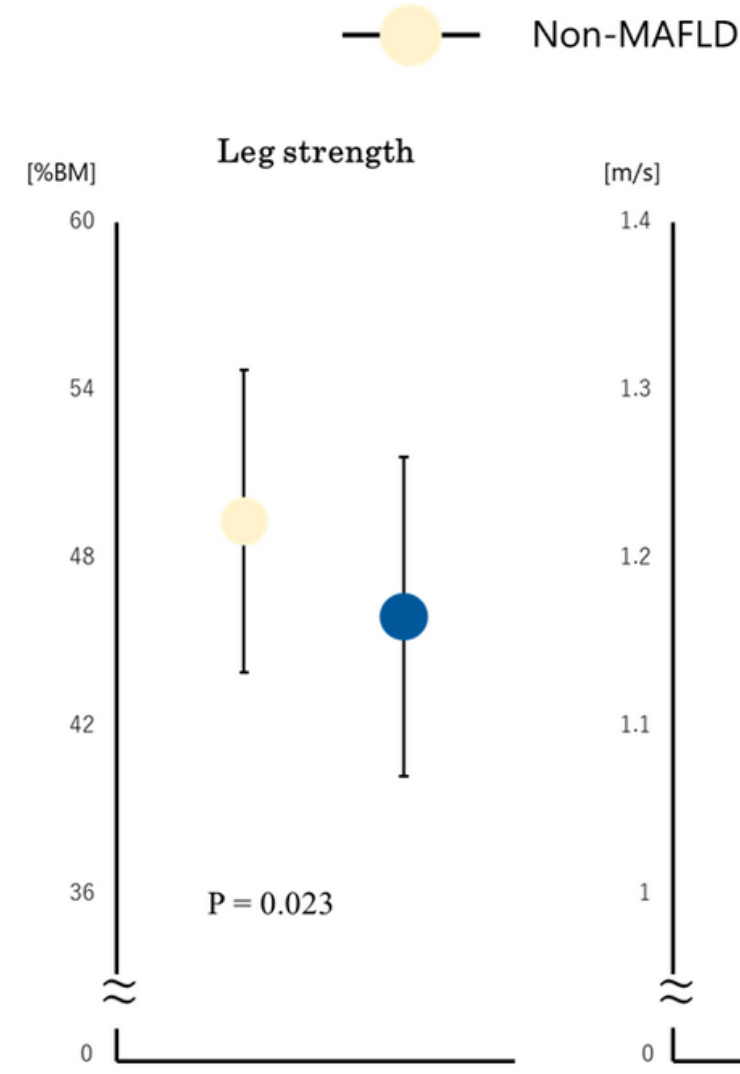

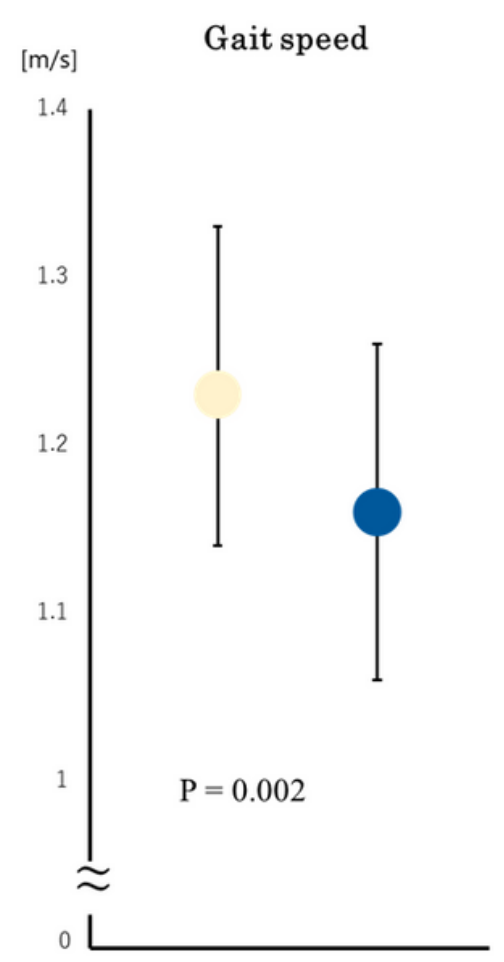

MAFLD
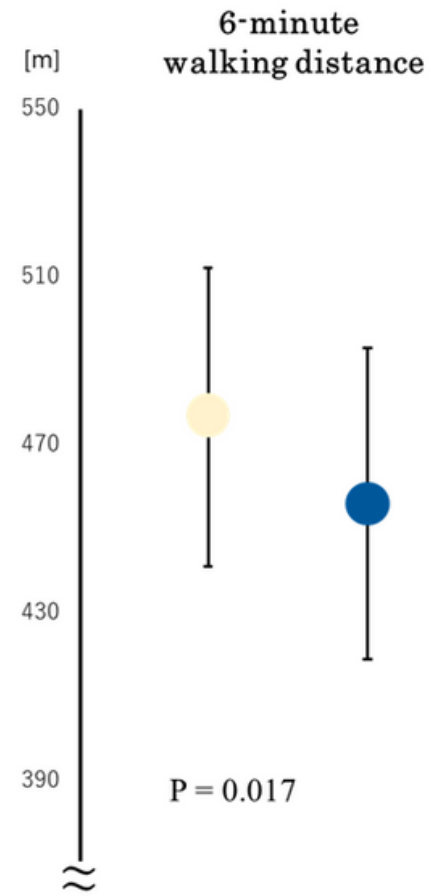

0

\section{Figure 3}

Multiple regression models of MAFLD with leg strength, gait speed, and 6MWD. Estimated mean values of leg strength, gait speed, and 6MWD in multiple regression models were adjusted for age, sex, BMI, log maximum CK, number of diseased vessels, statin, aspirin, prior heart failure, hypertension, dyslipidemia, diabetes mellitus, smoking, log albumin, log hemoglobin

MAFLD, metabolic dysfunction-associated fatty liver disease; 6MWD, 6-minute walking distance; BMI, body mass index; CK, creatine kinase 
MAFLD

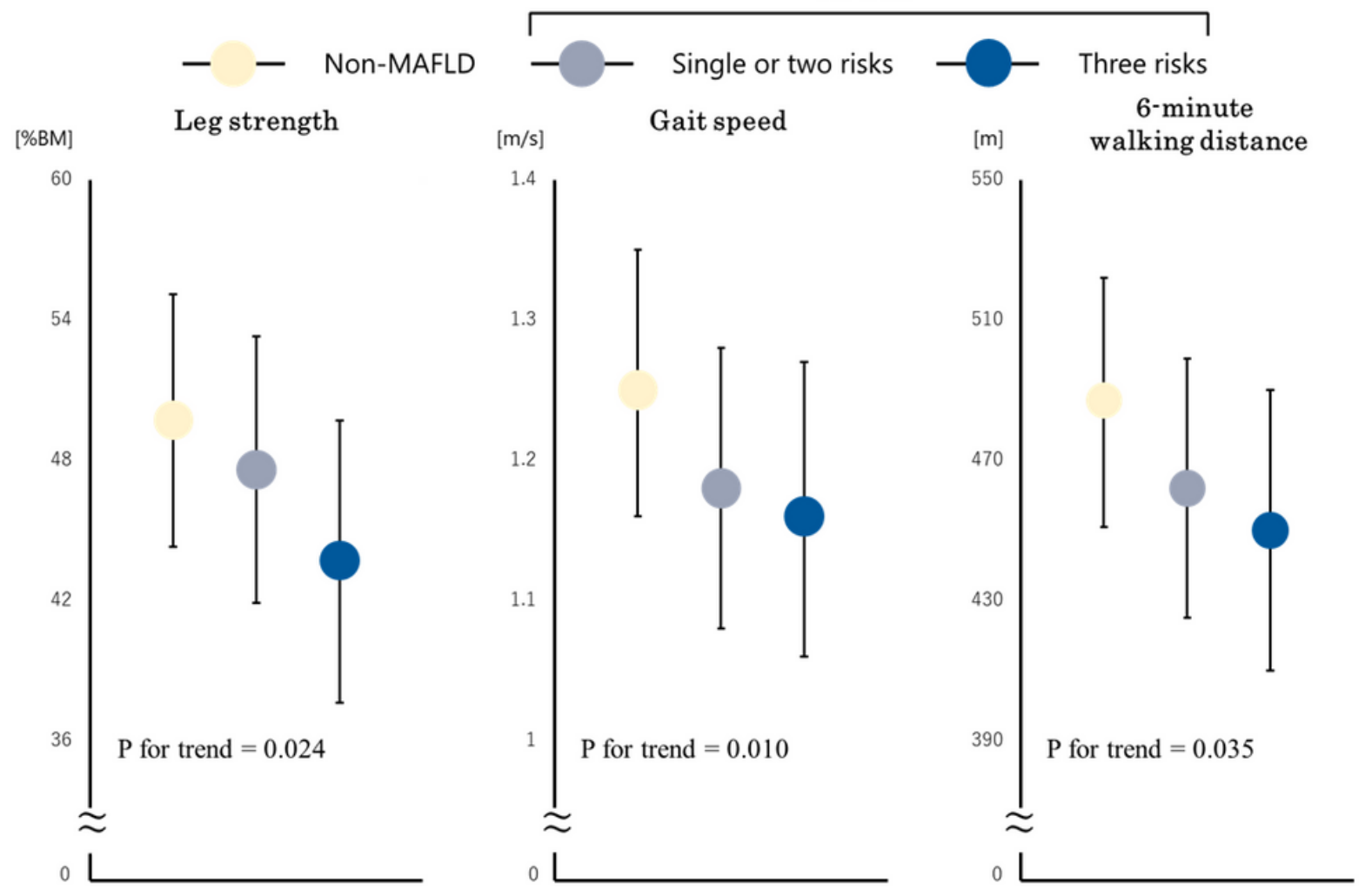

Figure 4

Multiple regression models of multiple metabolic condition disorders with leg strength, gait speed, and 6MWD. Estimated mean values of leg strength, gait speed, and 6MWD in multiple regression models were adjusted for age, sex, BMI, log maximum CK, number of diseased vessels, statin, aspirin, prior heart failure, hypertension, dyslipidemia, diabetes mellitus, smoking, log albumin, log hemoglobin

MAFLD, metabolic dysfunction-associated fatty liver disease; 6MWD, 6-minute walking distance; BMI, body mass index; CK, creatine kinase 